\title{
A endogamia explicaria a elevada prevalência de deficiências em populações do Nordeste brasileiro?
}

\author{
Could endogamy explain the higher prevalence of disabilities \\ in the population of the Brazilian Northeast?
}

Silvana Cristina dos Santos

Uirá Souto Melo ${ }^{1}$

Simone Silva dos Santos Lopes ${ }^{1}$

Mathias Weller ${ }^{1}$

Fernando Kok ${ }^{2}$

Departamento de Biologia

Abstract Despite conceptual inaccuracies and methods of measurement, the WHO estimates that about $10 \%$ of the world population has some form of disability. In order to investigate the prevalence and etiology of disabilities and to evaluate if they could be associated with inbreeding, a cross-sectional epidemiological study was conducted using the informant method in five communities of the state Rio Grande do Norte in the Brazilian Northeast, in which consanguineous marriages frequencies varied between 9 and 32\%. The average prevalence of disabilities in the five sampled communities was $4.53 \%$, obtained by interviews that involved $37.87 \%$ of a population of 39,054 inhabitants. On average, $25 \%$ of consanguineous and $12 \%$ of non-consanguineous couples had one or more children with disabilities. The increased rate of individuals with disabilities in the Brazilian Northeast could be associated with the maintenance of the tradition of consanguineous marriages in these populations and some of these disabilities may be caused by genetic disorders.

Key words Disability, Epidemiology, Brazil's northeast, Endogamy
Universidade Estadual da

Paraíba. Av. Baraúnas 351, Bairro Universitário. 58109-753 Campina Grande PB.

silvanaipe@gmail.com

${ }^{2}$ Hospital das Clínicas,

Universidade de São Paulo.
Resumo Apesar da inexatidão conceitual e de métodos de aferição, a OMS estima que cerca de 10\% da população mundial apresente alguma forma de deficiência. Com a finalidade de investigar a prevalência e a etiologia das deficiências e verificar se elas estariam associadas à endogamia, foi realizado um estudo epidemiológico transversal com uso do método do informante envolvendo cinco municípios do Estado do Rio Grande do Norte, no Nordeste brasileiro; nos quais foram estimadas frequências de casamentos consanguíneos que variam de 9 a 32\%. A prevalência média estimada para deficiências nos cinco municípios amostrados foi de 4,53\%, obtida por meio de entrevistas envolvendo 37,87\% de uma população de 39.054 habitantes. Em média, 25\% dos casais consanguíneos e $12 \%$ dos não consanguíneos apresentaram um ou mais filhos com alguma deficiência. A elevada prevalência de pessoas com deficiência no Nordeste brasileiro pode ter associação com a manutenção da tradição de casamentos consanguíneos nessas populações e parte dessas deficiências pode ser causada por doenças genéticas.

Palavras-chave Deficiência, Epidemiologia, Nordeste brasileiro, Endogamia 


\section{Introdução}

Há poucos estudos sobre a prevalência de diferentes deficiências no mundo. A conceituação e a classificação das deficiências possuem importância prática, pois políticas públicas e de acesso a serviços específicos dependem da precisão da terminologia, dos métodos de aferição e da caracterização etiológica ${ }^{1-4}$. Em 2001, a OMS publicou a Classificação Internacional de Funcionalidade, Incapacidade e Saúde (CIF), na qual foi definida deficiência como alterações de função ou de estruturas do corpo humano ${ }^{5-6}$. Apesar das variações em relação às estimativas sobre a prevalência de deficiências ${ }^{7-10}$, a OMS estima que cerca de $10 \%$ da população mundial apresente alguma forma de deficiência ${ }^{11} \mathrm{e}$, deste total, apenas $3 \%$ das pessoas que necessitam de reabilitação em países subdesenvolvidos recebem algum tipo de serviço ${ }^{12}$.

No Brasil, a distribuição das deficiências reflete em parte os padrões de pobreza. No censo demográfico do IBGE em 2000, as pessoas com deficiências e incapacidades representavam 14,5\% da população brasileira ${ }^{13} \mathrm{e}$ atingiram a fração de $23,9 \%$ na amostragem feita em $2010^{14}$. No documento "Retratos da Deficiência no Brasil"15 foi revelado que os estados com maior taxa de pessoas com deficiência e incapacidades se localizam no Nordeste: a Paraíba liderava o "ranking" com $18,76 \%$ de sua população com alguma deficiência, seguida pelo Rio Grande do Norte (17,64\%), Piauí (17,63\%), Pernambuco $(17,4 \%)$ e Ceará $(17,3 \%)$. Não se sabe ao certo se a pobreza efetivamente explicaria esses achados ${ }^{16}$, tendo em vista que não existem estudos que evidenciem isto. Outros fatores, de natureza ambiental, como infecções, traumas e violência; ou genéticos, como a endogamia; ou ainda socioeconômicos, como desnutrição materna e processos migratórios, poderiam explicar a maior porcentagem de deficiência nestas regiões. Estudos recentes, por exemplo, mostraram que municípios do sertão do Estado do Rio Grande do Norte possuem algumas deficiências causadas por doenças genéticas de herança recessiva associadas à endogamia ${ }^{17-18}$.

No presente trabalho, são descritos resultados de estudo sobre prevalência de deficiências causadas ou não por doenças genéticas, realizado em cinco municípios no Nordeste brasileiro. Esses municípios apresentam taxas de casamentos consanguíneos que variam de 9 a $32 \%{ }^{19}$. A intenção foi criar categorias de classificação que pudessem revelar, com mais detalhes, quais são as deficiências que acometem as populações amostradas e sua provável etiologia (ambiental ou genética). E, além disso, mostrar a relação entre deficiência e endogamia. A classificação das deficiências foi realizada em 31 categorias organizadas em cinco subgrupos que agrupam descrições de graus de perda de função dos pacientes em relação a alguma capacidade, quer seja motora ou intelectual.

\section{Métodos}

\section{Amostra e Procedimentos}

O levantamento das doenças genéticas e de outras deficiências de interesse médico-sanitário foi realizado em cinco municípios do Estado do Rio Grande do Norte, os quais se encontram listados entre os 50 municípios brasileiros com maior número de portadores de deficiências ${ }^{15}$. Esses municípios estão localizados em áreas rurais, relativamente isoladas, distando $350-400 \mathrm{~km}$ da capital do estado, Natal. A população total envolvida nesta pesquisa, segundo estimativa do Censo 2007, é de 39.054 habitantes, distribuídos nos seguintes municípios: Serrinha dos Pintos com 4.360 habitantes; Riacho de Santana com 4.292; Pilões com 3.381; Olho d'Água do Borges com 4.442 e São Miguel com 22.579.

A coleta de dados sobre a ocorrência de deficiências e sua relação com casamentos consanguíneos foi realizada pelos agentes comunitários do "Programa de Agentes Comunitários de Saúde (PACS)" orientados pela equipe de enfermeiros do "Programa de Saúde da Família" (PSF) e pelos pesquisadores envolvidos neste trabalho. Em um curso de formação de dois dias realizado em cada um dos municípios participantes separadamente, os agentes e enfermeiros foram instruídos a entrevistar os casais residentes em suas comunidades e a preencher uma ficha com dados sobre o grau de parentesco do casal, seu número de filhos, a presença ou não de indivíduos portadores de deficiências ou incapacidades (Ficha CO). Após a coleta de dados em caráter de estudo-piloto, os agentes participaram de um novo encontro para esclarecimentos e correções nos procedimentos de campo.

A relação de parentesco dos casais foi obtida por meio da declaração feita por um dos seus membros entrevistado pelo agente de saúde. Essa relação foi classificada em: não consanguínea (NC); tio-sobrinha (TS) ou primos duplos em $1^{\circ}$ grau $(\mathrm{PD})$; primos em $1^{\circ}$ grau $(\mathrm{PL})$; primos de $2^{\circ}$ grau (P2); primos em $3^{\circ}$ grau (P3); parentesco mais distante do que primos em $2^{\circ}$ grau ou 
parentesco existente, mas não classificado $(\mathrm{P}+)$. A validação dos dados foi realizada em um dos municípios (Pilões) onde 12,5\% das famílias amostradas foram revisitadas e novamente entrevistadas por um dos pesquisadores (SS). Foram identificadas as seguintes incorreções no preenchimento das fichas: três erros na classificação de grau de parentesco; 12 erros em relação à idade ou nome de um dos membros do casal (isso ocorreu nas famílias mais idosas nas quais os próprios depoentes têm dificuldade de informar corretamente o dado solicitado); 25 erros na classificação das deficiências e 12 que não mencionaram uma determinada anomalia em virtude da omissão dos próprios informantes. A estimativa da porcentagem total de erros ocorridos durante o preenchimento das fichas foi da ordem de $3 \%$.

Todas as atividades foram realizadas após a devida avaliação e aprovação do Comitê de Ética em Pesquisas com Seres Humanos do Instituto de Biociências da Universidade de São Paulo.

\section{Classificação das deficiências}

A classificação das deficiências foi realizada em 31 categorias organizadas em cinco subgrupos que reúnem descrições de graus de perda de função dos pacientes em relação a alguma capacidade, quer seja física ou intelectual. Essa classificação foi criada a partir da adaptação das categorias utilizadas pelo IBGE: grande dificuldade (GE) ou incapacidade (IE) para enxergar; grande dificuldade (GC) ou incapacidade (IC) para caminhar; grande dificuldade (GO) ou incapacidade (IO) para ouvir; com deficiência mental (IM) ou dificuldade para aprender (DA); falta de membros (IF); paralisia das pernas (IP), de um dos lados do corpo (IL) ou total (IT); incapacidades ou deficiências múltiplas (IMU); distúrbios psiquiátricos (DP).

As subdivisões das deficiências utilizadas neste trabalho, descritas juntamente com os resultados (Tabela 1), buscaram diferenciar as deficiências que são congênitas daquelas que não são; as que são progressivas das que não são; e aquelas que acarretam deformidades. As descrições das deficiências visuais e auditivas mantiveram a subdivisão entre dificuldade e incapacidade, incorporando a informação de congênito ou não. No caso das deficiências físicas ou motoras, as subdivisões abrangem a dificuldade ou a incapacidade para andar devido à perda de força característica das distrofias musculares; da perda de coordenação ou movimento, característica das doenças neurodegenerativas. Além disso, discrimina as pessoas que não andam porque sofreram acidentes; porque têm defeitos congênitos ou escoliose.

No grupo das deficiências de natureza psiquiátrica ou neurológica foram reunidas as dificuldades de aprendizagem; pessoas que apresentam convulsão e epilepsia; deficiência intelectual (ou retardo mental) e distúrbios psiquiátricos discriminados em relação à sua gravidade bem como pessoas que utilizam benzodiazepínicos. As síndromes genéticas e as malformações constituíram outro subgrupo. Essas categorias de classificação constituem aproximações para melhor caracterizar as deficiências que atingem as populações e foram criados a partir das observações empíricas realizadas pelos pesquisadores envolvidos neste estudo.

A análise descritiva e estatística foi realizada com software PASW STATISTICS, versão 17 (SPSS Inc., IBM Company). As médias das frequências de casais consanguíneos e não consanguíneos com prole deficiente foram comparadas usando teste de Mann Whitney, adotando-se nível de significância de 5\%.

\section{Resultados e discussão}

A prevalência média estimada para deficiências nos cinco municípios amostrados neste trabalho foi de $4,53 \%$, tendo sido obtida por meio de entrevistas envolvendo $37,87 \%$ de uma população de 39.054 habitantes (Tabela 2). Os valores variaram de 1,26\% em Olho d'Água do Borges a 16,60\% em Serrinha dos Pintos. Neste município, a elevada taxa de deficientes poderia ser explicada pelo fato dos agentes terem amostrado praticamente o dobro da população em relação aos outros municípios. Por um lado, Serrinha pode realmente ter uma prevalência maior de pessoas com deficiência, tendo em vista seu isolamento e as taxas de endogamia. Por outro lado, a capacitação oferecida aos profissionais da área da saúde durante a realização desta pesquisa pode não ter sido suficiente para padronizar os procedimentos de classificação em todos os municípios envolvidos, pois ela foi realizada em cada um deles separadamente.

Dependendo do entendimento do responsável pela coordenação do levantamento de dados no município, os critérios de inclusão podem ter variado. Algumas equipes, como em Serrinha dos Pintos, levantaram informações sobre todas as pessoas que apresentavam alguma forma de de- 
Tabela 1. Classificação e distribuição das diferentes deficiências em cada um dos municípios amostrados do estado do Rio Grande do Norte. (RS: Riacho de Santana; OB: Olho d’Água do Borges; PI: Pilões; SM: São Miguel; SP: Serrinha dos Pintos). $\mathbf{N}$ refere-se ao total de pessoas com deficiências encontradas no município.

\begin{tabular}{|c|c|c|c|c|c|c|}
\hline & Categorias para classificação das deficiências & $\begin{array}{c}\mathrm{RS} \\
\mathrm{N}=152 \\
\%\end{array}$ & $\begin{array}{c}\mathrm{OB} \\
\mathrm{N}=56 \\
\%\end{array}$ & $\begin{array}{c}\mathrm{PI} \\
\mathrm{N}=105 \\
\%\end{array}$ & $\begin{array}{c}\mathrm{SM} \\
\mathrm{N}=734 \\
\%\end{array}$ & $\begin{array}{c}\mathrm{SP} \\
\mathrm{N}=724 \\
\%\end{array}$ \\
\hline DA & 1. Dificuldade para enxergar desde o nascimento & 3,95 & 0 & 2,86 & 4,5 & 8,15 \\
\hline DA & 2. Perda progressiva, mas ainda vê & 9,2 & 1,75 & 4,76 & 3,95 & 19,75 \\
\hline DA & 3. Não enxerga desde o nascimento & 0 & 5,35 & 0,95 & 1,36 & 0,41 \\
\hline DA & 4. Perda progressiva até cegueira & 0 & 3,57 & 1,9 & 1,09 & 0,14 \\
\hline DV & 5. Dificuldade para ouvir desde o nascimento & 4,6 & 0 & 0,95 & 2,04 & 2,62 \\
\hline DV & 6. Perda progressiva da audição, mas ainda ouve & 1,32 & 0 & 0,95 & 1,09 & 0,83 \\
\hline DV & $\begin{array}{l}\text { 7. Incapacidade para ouvir e falar desde o nascimento } \\
\text { (surdo-mudo) }\end{array}$ & 5,92 & 10,69 & 1,9 & 5,04 & 0,97 \\
\hline DV & 8. Perda progressiva da audição (não ouve, mas fala) & 0,66 & 0 & 0 & 0,27 & 0,28 \\
\hline DV & 9. Perda de equilíbrio ou coordenação & 0,66 & 0 & 0 & 1,79 & 0,29 \\
\hline $\mathrm{DM}$ & $\begin{array}{l}\text { 10. Perna mais curta, pé é torto e/ou deformação de } \\
\text { membros }\end{array}$ & 9,2 & 12,4 & 4,76 & 6,4 & 5,26 \\
\hline $\mathrm{DM}$ & 11. Pés são tortos com pernas atrofiadas & 1,32 & 3,54 & 0 & 2,72 & 1,8 \\
\hline $\mathrm{DM}$ & 12. Não move os membros facilmente, anda mancando & 0,66 & 1,75 & 0 & 0,27 & 0,28 \\
\hline $\mathrm{DM}$ & 13. Perdeu a força, mas pé e perna se movem & 2,63 & 1,78 & 0 & 1,23 & 0,55 \\
\hline $\mathrm{DM}$ & 14. Problema na coluna (escoliose ou sifose) & 1,97 & 7,15 & 1,9 & 7,77 & 16,03 \\
\hline $\mathrm{DM}$ & 15. Nunca andou, incapacidade desde o nascimento & 0,66 & 1,79 & 1,9 & 2,32 & 0,69 \\
\hline $\mathrm{DM}$ & $\begin{array}{l}\text { 16. Andou mas perdeu progressivamente essa capacidade } \\
\text { até ficar na cadeira de rodas }\end{array}$ & 0 & 0 & 0,95 & 1,5 & 0,98 \\
\hline $\mathrm{DM}$ & 17. Não anda, porque nasceu com ausência de membros & 0 & 0 & 0 & 0 & 0 \\
\hline $\mathrm{DM}$ & $\begin{array}{l}\text { 18. Não anda porque sofreu acidente (veículo, trabalho, } \\
\text { residência) }\end{array}$ & 0,66 & 0 & 0 & 0,54 & 0,69 \\
\hline $\mathrm{DP}$ & 19. Duas ou mais crises convulsivas & 7,89 & 1,79 & 10,5 & 7,36 & 7,75 \\
\hline $\mathrm{DP}$ & $\begin{array}{l}\text { 20. Deficiência intelectual: desde a infância, a pessoa é } \\
\text { "calada", não aprendeu a fazer higiene pessoal ou tarefas } \\
\text { domésticas }\end{array}$ & 6,58 & 12,7 & 5,71 & 3,68 & 0,98 \\
\hline DP & $\begin{array}{l}\text { 21. Tem entendimento, mas não aprende facilmente ou } \\
\text { é hiperativo }\end{array}$ & 3,95 & 5,35 & 9,52 & 1,77 & 3,9 \\
\hline $\mathrm{DP}$ & $\begin{array}{l}\text { 22. Distúrbio psiquiátrico grave (com episódios de } \\
\text { internação hospitar ou no CAPS) }\end{array}$ & 3,29 & 5,35 & 6,67 & 7,77 & 4,7 \\
\hline DP & $\begin{array}{l}\text { 23. Distúrbio psiquiátrico moderado (surtos, delírio ou } \\
\text { crises de variação de humor) }\end{array}$ & 4,61 & 0 & 0 & 6,54 & 1,24 \\
\hline $\mathrm{DP}$ & $\begin{array}{l}\text { 24. Usuário de benzodiazepínicos (drogas com uso } \\
\text { controlado usados para depressão e para dormir) }\end{array}$ & 14,47 & 14,29 & 32,38 & 17,17 & 12,75 \\
\hline $\mathrm{DP}$ & $\begin{array}{l}\text { 25. Desde o nascimento com deficiências múltiplas (não } \\
\text { anda, não fala ou/ou não ouve, entende pouco) }\end{array}$ & 0,66 & 0 & 0 & 1,09 & 0,69 \\
\hline $\mathrm{DP}$ & 26. Alterações de comportamento (autismo) & 0,66 & 1,79 & 0 & 0,68 & 0,3 \\
\hline DG & $\begin{array}{l}\text { 27. Malformações ao nascimento (crânio, face, lábio, } \\
\text { orelhas, membros) }\end{array}$ & 2,63 & 1,79 & 0,95 & 1,23 & 1,27 \\
\hline $\mathrm{DG}$ & $\begin{array}{l}\text { 28. Malformações em mãos e pés: dedos a mais ou a } \\
\text { menos; mais curtos; com inserção diferente do normal } \\
\text { ou grudados }\end{array}$ & 4,61 & 3,59 & 4,77 & 2,86 & 1,9 \\
\hline DG & $\begin{array}{l}\text { 29. Doença ou síndrome genética diagnosticada por } \\
\text { médico }\end{array}$ & 1,32 & 0 & 0 & 1,23 & 0,92 \\
\hline DG & $\begin{array}{l}\text { 30. Diagnosticado como afetado pela Síndrome SPOAN } \\
\text { (doença neurogenetiva) }\end{array}$ & 0 & 1,79 & 0 & 1,08 & 2,64 \\
\hline \multirow[t]{2}{*}{ DG } & $\begin{array}{l}\text { 31. Síndrome de Down: deficiência mental com face } \\
\text { característica }\end{array}$ & 5,92 & 1,79 & 5,72 & 3,68 & 1,52 \\
\hline & Total & 100 & 100 & 100 & 100 & 100 \\
\hline
\end{tabular}


ficiência, incapacitante ou não, com o máximo de detalhamento e abrangência possível. Essa dificuldade em relação à padronização de procedimentos para a inclusão e a exclusão de pessoas como deficiente já foi extensamente debatido na literatura; tendo sido, por exemplo, criadas duas categorias de classificação pelo IBGE para sanar em parte esse desafio: pessoas portadoras de deficiência (PPD) e pessoas portadoras de incapacidade $(\mathrm{PPI})^{13}$.

Essa variação na porcentagem de pessoas com deficiência encontrada em nosso estudo também pode ser observada quando comparadas as estimativas disponíveis no banco de dados do SIAB/ DATASUS ${ }^{20}$ e no documento "Retratos da Deficiência no Brasil” do IBGE. As estimativas de deficiência disponibilizadas pelo SIAB em 2008 estão abaixo de 5\% e os valores são da mesma ordem de grandeza daqueles obtidos neste levantamento. Entretanto, os dados do IBGE mostram valores maiores tanto para as deficiências incapacitantes (de 5 a 10\%) quanto para as não incapacitantes (30 a 35\%).

Essas diferenças ocorreram porque não existem métodos padronizados para classificação das deficiências e aferição de dados de prevalência. Na base de dados do SIAB, as informações colhidas mensalmente pelos agentes comunitários de saúde referem-se às deficiências provavelmente incapacitantes. Os estudos feitos pelo IBGE e outros bancos de informações, como dados da previdência social, usados para produção do documento "Retratos da Deficiência no Brasil", abrangem um conjunto maior de deficiências, inclusive aquelas de natureza psiquiátrica e neurológica. Neste caso, por exemplo, foram

Tabela 2. Porcentagem de pessoas com deficiências segundo levantamento feito por agentes de saúde em cinco municípios do estado do Rio Grande do Norte (RS: Riacho de Santana; OB: Olho d'Água do Borges; PI: Pilões; SM: São Miguel; SP: Serrinha dos Pintos; N: Número de pessoas com deficiência no município).

\begin{tabular}{lrrr}
\hline $\begin{array}{c}\text { Municípios } \\
\text { do Rio Grande } \\
\text { do Norte }\end{array}$ & $\begin{array}{c}\text { População } \\
\text { (IBGE }\end{array}$ & $\begin{array}{c}\text { Popop) } \\
\text { Amostracão }\end{array}$ & $\begin{array}{c}\text { Pessoas } \\
\text { com } \\
\text { Deficiência }\end{array}$ \\
\hline RS (N=152) & 4.292 & $44,92 \%$ & $3,54 \%$ \\
OB (N=56) & 4.442 & $33,18 \%$ & $1,26 \%$ \\
PI (N=105) & 3.381 & $44,66 \%$ & $3,11 \%$ \\
SM (N=734) & 22.579 & $31,68 \%$ & $3,25 \%$ \\
SP (N=724) & 4.360 & $62,48 \%$ & $16,60 \%$ \\
Total $(\mathrm{N}=1.771)$ & 39.054 & $37,87 \%$ & $4,53 \%$ \\
\hline
\end{tabular}

incluídas pessoas que recebem benefício por apresentarem distúrbios psiquiátricos que não necessariamente são vistas como deficiente pelos agentes de saúde.

\section{Distribuição das deficiências}

As deficiências foram agrupadas em cinco grupos: 1) deficiência auditiva (DA);2) deficiência visual (DV); 3 ) deficiência física ou motora (DM); 4) deficiências de natureza psiquiátrica e neurológica agrupando pessoas com epilepsia, deficiência intelectual, distúrbios psiquiátricos e dependência química (DP); 5) síndromes genéticas e malformações congênitas (DG). O Gráfico 1 apresenta a distribuição desses diferentes grupos nos municípios amostrados, explicitando a heterogeneidade da ocorrência dessas deficiências.

Cerca de $60 \%$ das pessoas com deficiências em Pilões apresentam epilepsia, diferentes distúrbios psiquiátricos e deficiência intelectual e, no restante dos municípios, os valores desse grupo de doenças psiquiatrias e neurológicas atingem $40 \%$ da totalidade das deficiências. A elevada frequência de pessoas com distúrbios psiquiátricos pode estar associada à cultura de consumo de benzodiazepínicos. Considerando alguns relatos dos profissionais da área de saúde, é muito comum a automedicação entre familiares. Geralmente, uma pessoa inicia um tratamento médico ingerindo tranquilizantes e ansiolíticos, e ela ofe-

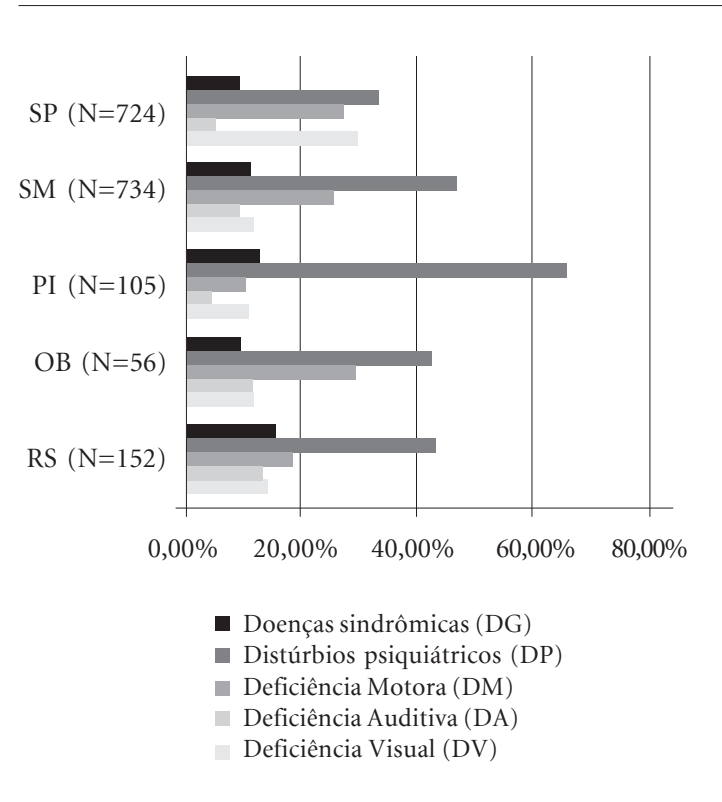

Gráfico 1. Distribuição de grupos de deficiências nos municípios avaliados. 
rece comumente a medicação aos seus familiares que passam a usá-la rotineiramente sem a devida orientação. Com o tempo, essas pessoas podem apresentar quadro de dependência química. A cultura da automedicação poderia explicar essa elevada frequência de pessoas com transtornos psiquiátricos.

Por outro lado, o município de Pilões também apresenta menor porcentagem de pessoas com deficiência auditiva e motora. Em relação à deficiência visual, Serrinha dos Pintos apresenta quase o dobro de casos quando comparada aos outros municípios. A porcentagem de deficiência motora atinge cerca de $20 \%$ das populações avaliadas, sendo maior em Serrinha dos Pintos, São Miguel e Olho d'Água do Borges. Nos dois primeiros foram encontradas aproximadamente 60 pessoas afetadas por uma doença neurodegenerativa de herança autossômica recessiva, a síndrome Spoan ${ }^{17,18}$. Isto não foi observado no terceiro município citado, onde ocorrem muitos casos de poliomielite.

Em estudos realizados na Europa ${ }^{8}$ e Oriente Médio $^{11,12}$, o padrão de distribuição de deficiências mostra maior porcentagem de deficientes físicos com dificuldade ou incapacidade de locomoção com média de 35\%, seguida pela deficiência intelectual (16\%) e da fala $(11,4 \%)$, o que difere das comunidades brasileiras analisadas com uma média de $40 \%$ de distúrbios psiquiátricos, epilepsia e deficiência intelectual. Essas diferenças podem ser explicadas em virtude dos distintos ambientes socioeconômico-culturais. Em algumas localidades, há uma proporção maior de deficientes físicos devido à violência e guerras civis, como ocorre em algumas regiões do Oriente Médio.

A caracterização de cada um dos grupos de deficiência para a obtenção de uma descrição mais precisa que pudesse indicar sua etiologia e progressão foi realizada por meio da classificação das deficiências em 31 categorias descritivas, que foram sintetizadas na Tabela 1 , bem como a porcentagem da população de deficientes agrupada em cada uma dessas categorias.

As distintas formas de deficiência visual ocorrem em diferentes proporções nos municípios. As deficiências congênitas abrangem cerca de $40 \%$ da amostra enquanto as perdas progressivas são mais frequentes; a maior parte das pessoas perde paulatinamente a capacidade de enxergar. Esse padrão de perda progressiva não foi verificado para as deficiências auditivas, pois muitas pessoas investigadas apresentam surdez congênita ("surdo e mudo"). Essas deficiências congênitas podem ser causadas tanto por mutações genéticas transmissíveis ao longo das gerações quanto por infecções, como rubéola, que atingem a criança durante o período pré-natal. As deficiências auditivas causadas por fatores ambientais normalmente não atingem o indivíduo simetricamente ou são adquiridas ao longo da vida.

Em relação às deficiências motoras, foi observado que elas atingem de $9,5 \%$ a $28,4 \%$ da totalidade da amostra e há uma elevada frequência da categoria 10, ou seja, de indivíduos que apresentam uma perna maior do que outra. $\mathrm{Na}$ maior parte dos casos, essa assimetria é devido à sequela de poliomielite, doença muito comum na região até vinte anos atrás quando foi erradicada. Outra deficiência muito frequente é a escoliose e deformidades de coluna e tronco, que acarretam também em dificuldade de locomoção. Em Serrinha dos Pintos, foram identificados 18 indivíduos que andavam durante a infância e perderam progressivamente a capacidade de andar e depois foram diagnosticados como afetados pela síndrome Spoan. Pouquíssimas pessoas nasceram com malformações em membros.

No grande grupo das deficiências de natureza psiquiátrica e neurológica foram reunidas tanto as pessoas com deficiência intelectual (retardo mental) quanto aquelas com transtornos psiquiátricos. Aproximadamente $20 \%$ dessas dificuldades que afetam o sistema nervoso são causadas por convulsão ou epilepsia. Em todas as localidades, cerca de $10 \%$ dessas deficiências são distúrbios psiquiátricos graves, cujos afetados precisaram de internação hospitalar. A dependência de benzodiazepínicos também é bastante séria, atingindo 32\% da população de Pilões e aproximadamente 15\% das demais populações. As deficiências intelectuais (retardo mental) atingem 1\% em Serrinha dos Pintos a 13\% em Olho d'Água do Borges. Essa variação novamente observada na classificação dessas diferentes formas de deficiência deve estar associada às definições que demandam conhecimentos técnicos específicos.

Independentemente das dificuldades de classificação, verifica-se que os problemas relacionados à saúde mental são os que atingem em maior proporção as populações que vivem no sertão do estado do Rio Grande do Norte e ações públicas específicas para entender esse fenômeno devem ser fortemente estimuladas. Certamente, uma hipótese para explicar essa situação é o consumo de benzodiazepínicos.

Em relação às doenças sindrômicas ou que acarretam malformações no crânio ou face como lábio leporino ou craniossinostose, ou mesmo 
aquelas que acarretam poli ou oligodactilia, ou ainda síndromes conhecidas e diagnosticadas, como as síndromes Spoan ou Down, foi verificado que de $8 \%$ a $14 \%$ das deficiências foram classificadas nestas categorias conforme mostra a Tabela 1 .

A distribuição das diferentes formas de deficiência sindrômica mostra que em Pilões e Riacho de Santana não foram encontrados indivíduos afetados pela síndrome Spoan. No caso de Olho d'Água do Borges possivelmente as pessoas com deficiência motora foram erroneamente classificados devido à interpretação equivocada dos agentes de saúde. Nas palestras oferecidas pelos pesquisadores envolvidos nesta pesquisa, foram mostrados exemplos com afetados pela síndrome Spoan e isto pode ter influenciado os agentes já que nenhum caso foi identificado no município. Em Serrinha dos Pintos e São Miguel, a proporção de indivíduos está adequada correspondendo ao número efetivo de casos diagnosticados.

\section{Deficiência e Endogamia}

Em média 25,5\% dos casais consanguíneos e 12,2\% dos não consanguíneos apresentaram um ou mais filhos com alguma deficiência (Tabela 3). Em Serrinha dos Pintos, um terço dos casais é consanguíneo e lá 35,2\% desses casais possuem ao menos um filho com deficiência. Essa porcentagem de prole deficiente se reduz para 7,4\% em Olho d'Água do Borges, que está situada nas margens de uma rodovia e apresenta maior mobilidade populacional e menor consanguinidade (9\%). Entre todos os casais aparentados amostrados, 1/3 aproximadamente apresentam filho deficiente e essa proporção se reduz para 1/7 entre os casais não aparentados.

Os casais consanguíneos apresentam em média maior porcentagem de filhos deficientes do que os não aparentados. A análise estatística mostrou que essa diferença entre os dois grupos é significativa quando se aplica o teste de Mann Whitney ( $p=0,041)$. Em média, 5,4\% dos filhos de casais não consanguíneos possuem alguma deficiência e esse valor praticamente é duplicado entre casais consanguíneos (10,5\%). Um em cada nove filhos de casais aparentados é deficiente, enquanto essa proporção se reduz para 1/17 entre os filhos de casais não aparentados (Tabela 3). Esses dados indicam claramente que os casais aparentados apresentam mais filhos com deficiência, e uma parte dessas deficiências deve ser

Tabela 3. Porcentagem de casais com prole normal (PN) e com prole deficiente (PD); e porcentagem de filhos normais (PN) ou com alguma deficiência (PD) fruto de uniões não consanguíneas e consanguíneas. (N: número total de casais ou de filhos amostrados; N1: número de casais ou filhos de casais não consanguíneos; N2: número de casais ou filhos de casais consanguíneos; RS: Riacho de Santanta; OB: Olho d'Água do Borges; PI: Pilões; SM: São Miguel e SP: Serrinha dos Pintos).

\begin{tabular}{|c|c|c|c|c|c|c|c|c|c|c|c|c|c|c|c|c|c|}
\hline & \multicolumn{3}{|c|}{ Casais } & \multicolumn{7}{|c|}{ Casais não-consanguíneos (CNC) } & \multicolumn{7}{|c|}{ Casais consanguíneos (CC) } \\
\hline & \multirow[b]{2}{*}{$\begin{array}{c}\text { Total } \\
\mathrm{N}\end{array}$} & \multirow[b]{2}{*}{$\begin{array}{l}\mathrm{CNC} \\
\mathrm{N} 1\end{array}$} & \multirow[b]{2}{*}{$\begin{array}{l}\mathrm{CC} \\
\mathrm{N} 2\end{array}$} & \multicolumn{3}{|c|}{ PN } & \multicolumn{3}{|c|}{ PD } & \multirow[b]{2}{*}{$\begin{array}{c}\text { CNC } \\
\text { PN/PD }\end{array}$} & \multicolumn{3}{|c|}{ PN } & \multicolumn{3}{|c|}{ PD } & \multirow[b]{2}{*}{$\begin{array}{c}\text { CC } \\
\text { PN/PD }\end{array}$} \\
\hline & & & & $\mathbf{n}$ & $\begin{array}{l}\% \\
\mathrm{~N}\end{array}$ & $\begin{array}{c}\% \\
\text { N1 }\end{array}$ & $\mathbf{n}$ & $\begin{array}{l}\% \\
\mathrm{~N}\end{array}$ & $\begin{array}{c}\% \\
\text { N1 }\end{array}$ & & n & $\begin{array}{l}\% \\
\mathrm{~N}\end{array}$ & $\begin{array}{c}\% \\
\text { N2 }\end{array}$ & $\overline{\mathbf{n}}$ & $\begin{array}{l}\% \\
\mathrm{~N}\end{array}$ & $\begin{array}{c}\% \\
\mathrm{~N} 2\end{array}$ & \\
\hline RS & 945 & 764 & 181 & 689 & 72,9 & 90,2 & 75 & 7,9 & 9,8 & 9 & 141 & 14,9 & 77,9 & 40 & 4,2 & 22,1 & 4 \\
\hline OB & 2 & & 6 & 624 & 5,2 & 94,0 & 40 & 5,5 & 6,0 & 16 & 63 & 8,6 & 9 & 5 &, 7 & 7,4 & 13 \\
\hline PI & 739 & 646 & 93 & 579 & 78,3 & 89,6 & 67 & 9,1 & 10,4 & 9 & 73 & 9,9 & & 20 & 2,7 & 21,5 & 4 \\
\hline SM & 3449 & 2796 & 653 & 2473 & 71,7 & 88,4 & 323 & 9,4 & 11,6 & 8 & 505 & 14,6 & 77,3 & 148 & 4,3 & 22,7 & 5 \\
\hline SP & 1259 & 836 & 423 & 647 & 51,4 & 77,4 & 189 & 15,0 & 22,6 & 3 & 274 & 21,8 & 6 & 149 & 11,8 & 35,2 & 2 \\
\hline \multirow[t]{5}{*}{ Totais } & 7124 & 5706 & 1418 & 5012 & 70,4 & 87,8 & 694 & 9,7 & 12,2 & 7 & 1056 & 14,8 & 74,5 & 362 & 5,1 & 25,5 & 3 \\
\hline & \multicolumn{3}{|c|}{ Filhos } & \multicolumn{6}{|c|}{ Filhos de CNC } & & \multicolumn{7}{|c|}{ ilhos de CC } \\
\hline & \multirow{3}{*}{$\begin{array}{c}\text { Total } \\
\text { N }\end{array}$} & \multirow{3}{*}{$\begin{array}{l}\mathrm{CNC} \\
\mathrm{N} 1\end{array}$} & \multirow{3}{*}{$\begin{array}{l}\mathrm{CC} \\
\mathrm{N} 2\end{array}$} & \multicolumn{3}{|c|}{ PN } & \multicolumn{3}{|c|}{ PD } & \multirow{3}{*}{$\begin{array}{c}\text { CNC } \\
\text { PN/PD }\end{array}$} & \multicolumn{3}{|c|}{ PN } & \multicolumn{3}{|c|}{ PD } & \\
\hline & & & & $\mathbf{n}$ & $\%$ & $\%$ & $\mathbf{n}$ & $\%$ & $\%$ & & $\mathbf{n}$ & $\%$ & $\%$ & $\bar{n}$ & $\%$ & $\%$ & $\mathrm{CC}$ \\
\hline & & & & & $\mathbf{N}$ & N1 & & $\mathrm{N}$ & $\mathrm{N} 1$ & & & $\mathbf{N}$ & N2 & & $\mathbf{N}$ & $\mathrm{N} 2$ & $\mathrm{PN} / \mathrm{PD}$ \\
\hline RS & 58 & 2833 & 925 & 2735 & 72,8 & 96,5 & 98 & 2,6 & 3 , & 28 & 871 & 23,2 & 30,7 & 54 & 1,4 & 5,8 & 16 \\
\hline OB & 2529 & 2277 & 252 & 2227 & 88,1 & 97,8 & 50 & 2,0 & 2,2 & 45 & 246 & 9,7 & 10,8 & 6 & 0,2 & 2,4 & 41 \\
\hline PI & 2204 & 1870 & 334 & 1792 & 81,3 & 95,8 & 78 & 3,5 & 4, & & 307 & 13,9 & 16,4 & 27 & 1,2 & 8,1 & 11 \\
\hline SM & 13544 & 10392 & 3152 & 9912 & 73,2 & 95,4 & 480 & 3,5 & 4,6 & 21 & 2898 & 21,4 & 27,9 & 254 & 1,9 & 8,1 & 11 \\
\hline SP & 4697 & 2902 & 1795 & 2512 & 53,5 & 86,6 & 390 & 8,3 & 13,4 & 6 & 1461 & 31,1 & 50,3 & 334 & 7,1 & 18,6 & 4 \\
\hline Totais & 26732 & 20274 & 6458 & 19178 & 71,7 & 94,6 & 1096 & 4,1 & 5,4 & 17 & 5783 & 21,6 & 28,5 & 675 & 2,5 & 10,5 & 9 \\
\hline
\end{tabular}


causada por doenças genéticas. Na literatura, estima-se que a mortalidade de crianças de 0 a 10 anos de idade é 3,5\% maior entre a prole de casais consanguíneos ${ }^{21}$.

A análise da distribuição da prole deficiente em função do grau de parentesco do casal mostrou que em média 38,6\% dos deficientes são filhos de primos com grau de parentesco relativamente distante enquanto $34,1 \%$ são filhos de primos legítimos ou de primeiro grau. Menos de 4,6\% dos indivíduos deficientes são filhos de tio/ sobrinho ou primos carnais, ou seja, primos duplos em $1^{\circ}$ grau (Tabela 4).

No Gráfico 2, é mostrada a distribuição dos casais em relação à faixa etária, classificadas em quatro categorias (casais com menos de 20 anos; entre 20 e 49 anos; entre 50 e 70 anos e com mais de 70 anos). Observa-se claramente maior proporção de casais consanguíneos entre a faixa etária de 20-49 anos em relação aos casais não aparentados. Isto indica a conservação da tradição

Tabela 4. Distribuição de prole com alguma deficiência em função do grau de parentesco do casal.

\begin{tabular}{lrrrrr}
\hline \multicolumn{1}{c}{ Parentesco } & TS & PC & \multicolumn{1}{c}{ P1 } & \multicolumn{1}{c}{ P2 } & P3+ \\
\hline RS $(\mathrm{N}=50)$ & $0 \%$ & $4 \%$ & $24 \%$ & $26 \%$ & $46 \%$ \\
OB $(\mathrm{N}=4)$ & $0 \%$ & $0 \%$ & $25 \%$ & $0 \%$ & $75 \%$ \\
PI $(\mathrm{N}=27)$ & $0 \%$ & $3,7 \%$ & $33,3 \%$ & $29,6 \%$ & $33,3 \%$ \\
SM $(\mathrm{N}=237)$ & $3 \%$ & $8,9 \%$ & $40,1 \%$ & $16,9 \%$ & $31,2 \%$ \\
SP $(\mathrm{N}=263)$ & $1,5 \%$ & $1,1 \%$ & $30,8 \%$ & $22,8 \%$ & $43,7 \%$ \\
Total $(\mathrm{N}=581)$ & $1,9 \%$ & $4,6 \%$ & $34,1 \%$ & $20,8 \%$ & $38,6 \%$ \\
\hline
\end{tabular}

de casamentos consanguíneos entre os jovens adultos que ainda valorizam muito esse tipo de associação familiar. Essa manutenção da cultura de uniões entre pessoas aparentadas no Nordeste brasileiro ${ }^{22}$ contradiz um fenômeno mundial de mudança nos padrões reprodutivos, com redução significativa da endogamia.

Algumas pesquisas mostram redução na fertilidade entre casais consanguíneos devido ao compartilhamento de haplótipos específicos HLA, ou devido à expressão de genes deletérios durante a embriogênese e o desenvolvimento fetal, resultando em abortos espontâneos. Outras, entretanto, demonstram que a elevada compatibilidade genética entre mãe e feto durante a gravidez reduziria involuntariamente a esterilidade e as perdas prénatais. De maneira geral, verifica-se um aumento na fertilidade desses casais que pode ser explicada pela idade em que se casam e dão a luz ao primeiro filho, e pelo fato desses casais relutarem em utilizar contraceptivos ${ }^{23-25}$. Não se sabe ao certo se casais consanguíneos têm maior ou menor fertilidade em relação aos não aparentados.

Os efeitos prejudiciais das uniões consanguíneas são causados pela expressão de alelos recessivos e raros herdados de um ancestral comum ao casal. Nas populações onde este tipo de união é frequente, é esperado um aumento nos níveis de morbidade e mortalidade devido às mutações específicas encontradas em alguns locos gênicos. Esses alelos raros podem aumentar sua frequência na população quando ocorrem eventos de deriva genética ou efeito de fundador. Estudos

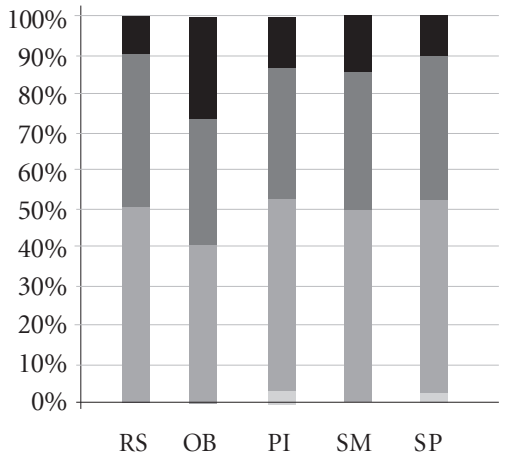

- $\mathrm{CNC}(\mathrm{N}=5.963)>70$

CNC $(\mathrm{N}=5.963) 50-70$

- CNC $(\mathrm{N}=5.963) 20-49$

-1 $\mathrm{CNC}(\mathrm{N}=5.960)<20$

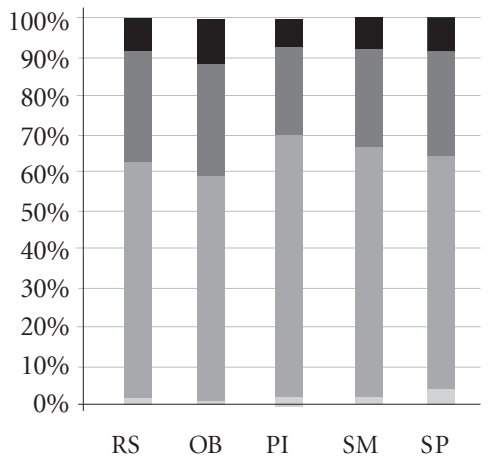

- $\mathrm{CNC}(\mathrm{N}=1.459)>70$

- CNC (N=1.459) 50-70

- CNC $(\mathrm{N}=1.459) 20-49$

- $\mathrm{CNC}(\mathrm{N}=1.459)<20$

Gráfico 2. Comparação entre a faixa etária de casais não consanguíneos (CNC) e consanguíneos (CC). 
empíricos sobre a prole de uniões entre primos em primeiro-grau indicam que os níveis de morbidade são de $1 \%$ a $4 \%$ maiores do que entre a prole da população em geral. Se, por um lado, existem estimativas de que a consanguinidade contribui em até $4 \%$ para aumentar a mortalidade em populações com coeficiente de endocruzamento de 0,0005 a 0,0370 , por outro, esses dados não podem ser analisados desconsiderando outros fatores de natureza socioeconômica ${ }^{26}$.

Espera-se um declínio na prevalência de consanguinidade em virtude da redução do tamanho das famílias. É provável que esse declínio não aconteça uniformemente nas populações, tendo em vista que nas cidades urbanizadas o nível de educação do casal e a idade de casamento são maiores do que em regiões menos desenvolvidas e rurais. Com a redução do tamanho das famílias, as uniões tio-sobrinha e entre primos duplos em primeiro grau dificilmente ocorrerão. Com a melhoria das condições socioeconômicas, a incidência de doenças "ambientais" em países em desenvolvimento se reduz em virtude da melhoria dos indicadores de saúde pú- blica, como atendimento pré-natal, vacinação, entre outros programas.

Segundo Bittles ${ }^{26,27}$, o conhecimento existente sobre consanguinidade tende a evidenciar os resultados clínicos negativos das uniões entre pessoas aparentadas que afetam uma minoria de famílias em países desenvolvidos. Entretanto, muito pouco é conhecido sobre a proporção de casais aparentados cujas crianças não possuem anomalias deletérias evidentes, e para quem não são mensurados os benefícios socioeconômicos que parecem óbvios. Para entender quais são os benefícios que preservam essas práticas em comunidades onde segregam doenças recessivas raras é necessário empreender pesquisas multidisciplinares para estimar a extensão desses problemas, acompanhadas de iniciativas de programas de aconselhamento genético comunitário. Face ao elevado número de casamentos consanguíneos existentes em muitos dos países populosos em desenvolvimento e ao fato de que muitas dessas doenças raras recessivas são letais, programas que tratem dessa temática trariam um benefício para a sociedade humana como um todo.

\section{Colaboradores}

SC Santos, US Melo, SSS Lopes, M Weller e F Kok participaram igualmente de todas as etapas de elaboração do artigo. 


\section{Referências}

1. Amiralian M, Pinto E, Ghirardi M, Lichtig I, Mansini E, Pasqualin L. Conceituando deficiência. Rev Saude Publica 2000; 34(1):97-103.

2. Brasil. Decreto no. 1.744, de dezembro, de 1995 Regulamenta o benefício de prestação continuada devido à pessoa portadora de deficiência e ao idoso. Diário Oficial da União 1999; 20 dez.

3. Diniz D, Squinca F, Medeiros M. Qual a deficiência? Perícia médica e assistência social no Brasil. Cad Saude Publica 2007; 23(11):2589-2596.

4. Atijosam O, Rischewsky D, Simms V, Kuper H, Linganwa B. A national survey of musculoskeletal impairment in Rwanda: prevalence, causes and service implications. Plos One 2008; 3(7):e2851-2857.

5. Portugal. Secretariado Nacional de Reabilitação. Classificação Internacional das Deficiências, Incapacidades e Desvantagens (Handicaps): Um manual de classificação das consequências das doenças (CIDID). Lisboa: SNR/OMS; 1989.

6. Organização Mundial da Saúde (OMS). CIF: classificação internacional de funcionalidade, incapacidade e saúde. São Paulo: Edusp; 2003.

7. Andresen EM, Prince-Caldwell A, Akinci F, Brownson CA, Haggund K, Jackson-Tompson J, Crocker R. The Missouri Disability Epidemiology and Health Project. Am J Prev Med 1999; 16(3):63-71.

8. Picavet HSJ, Hoeymans, N. Physical disability in The Netherlands: prevalence, risk groups and time trends. Public Health 2002; 116(4):231-237.

9. Chile. Fonadis. Primer Estudio Nacional de la Discapacidad en Chile [documento na internet] [acessado 2010 jul 7]. Disponível em: http://www.bcn.cl/ carpeta_temas/temas_portada.2005024.2908836766/ pdf/RESUMEN\%20ESTUDIO \%20NACIONAL\%20 DE\%20DISCAPACIDAD.pdf

10. Castro SS, César CLG, Carandina L, Barros MBA, Alves MCGP, Goldbaum M. Deficiência visual, auditiva e física: prevalência e fatores associados em estudo de base populacional. Cad Saude Publica 2008; 24(8):1773-1782.

11. World Health Organization (WHO). Disability and Rehabilitation: Future, Trends and Challenges in Rehabilitation. Geneva: WHO; 2002.

12. Handam M., Al-Akhras N. House-to-house survey of disabilities in rural communities in the north of the West Bank. East Mediterr Health J 2009; 15(6): 1496-1503.

13. Instituto Brasileiro de Geografia e Estatística (IBGE). Censo Demográfico 2000. Rio de Janeiro: IBGE; 2001

14. Instituto Brasileiro de Geografia e Estatística (IBGE). Censo Demográfico 2010: Resultados preliminares. [documento da Internet]. 2010. [acessado 2013 mar 20]. Disponível em: http://www.ibge.gov.br/home/ estatistica/populacao/censo2010/default.shtm

15. Néri M. Retratos da Deficiência no Brasil. Rio de Janeiro: FGV, IBRE, CPS; 2003.
16. Trani JF, Loeb M. Poverty and disability: a vicious circle? Evidence from Afghanistan and Zambia. J. Int. Dev. 2010; 24(Supl. 1):S19-S52. [serial on the Internet]. 2010. Jun. [cited 2011 Nov 9]. Available from: http://onlinelibrary.wiley.com/doi/10.1002/ jid.1709/pdf

17. Macedo-Souza LI, Kok F, Santos S, Amorim SC, Starling A, Nishimura A, Lezirovitz K, Lino AMM, Zatz M. Spastic Paraplegia, Optic Atrophy and Neuropathy (SPOAN Syndrome) is linked to chromosome 11q13. Annals of Neurology 2005; 57(5):730-737.

18. Macedo-Souza LI, Kok F, Santos S, Licinio L, Lezirovitz K, Cavaçana N, Bueno C, Amorim S, Pessoa A, Graciani Z, Ferreira A, Prazeres A, de Melo NA, Otto PA, Zatz M. Spastic Paraplegia, Optic Atrophy, and Neuropathy: New Observations, Locus Refinement, and Exclusion of Candidate Genes. Ann Hum Genet 2009; 73(Pt 3):382-387.

19. Santos S, Kok F, Weller M, Paiva FRL, Otto PA. Consanguinity in Northeastern Brazil: strategies for the prospection of new genetic disorders. Genetics and Molecular Biology 2010; 33(2):220-223.

20. Brasil. Ministério da Saúde (MS). SIAB: Sistema de Informação da Atenção Básica. [acessado 2012 jan 8]. Disponível em: http://www2.datasus.gov.br/SIAB /index.php

21. Bittles AH, Black ML. Consanguinity, human evolution, and complex diseases. PNAS 2010; 107(1) 1779-1786.

22. Freire-Maia N. Consanguinity marriages in Brazil. Rev. Bras. Biol. 1990; 50(4):863-866.

23. Bittles AH, Neel JV. The costs of human inbreeding and their implications for variations at the DNA level. Nat Genet 1994; 8(2):117-121.

24. Bittles AH, Makov E. Inbreeding in human populations: An assessment of the costs. In: Mascie-Tyalor CGN, Boyce AJ, organizadores. Human Mating Patterns. Cambridge: Cambridge University Press; 1988. p. 153-167.

25. 25. Bittles AH, Grant JC, Sullivan SG, Hussain R. Does inbreeding lead to increased human fertility? Ann Hum Biol 2002; 29(2):111-131.

26. Bittles AH. Endogamy, consanguinity and community genetics. J Genet 2002; 81(3):91-98.

27. Bittles AH. A community genetics perspective on consanguineous marriage. Commun Genet 2008; 11(6):324-330.

Artigo apresentado em 04/11/2011

Aprovado em 20/12/2011

Versão final apresentada em 09/01/2012 